\title{
Non-spherical source-surface model of the heliosphere: a scalar formulation
}

\author{
M. Schulz \\ Received: 19 September 1995 / Revised: 24 October 1996 / Accepted: 30 October 1996
}

Space Sciences Department, Lockheed Martin Palo Alto Research Laboratories, 3251 Hanover Street, Palo Alto, California 94304 (USA)

\begin{abstract}
The source-surface method offers an alternative to full MHD simulation of the heliosphere. It entails specification of a surface from which the solar wind flows normally outward along straight lines. Compatibility with MHD results requires this (source) surface to be non-spherical in general and prolate (aligned with the solar dipole axis) in prototypical axisymmetric cases. Mid-latitude features on the source surface thus map to significantly lower latitudes in the heliosphere. The model is usually implemented by deriving the $\mathbf{B}$ field (in the region surrounded by the source surface) from a scalar potential formally expanded in spherical harmonics, with coefficients chosen so as to minimize the mean-square tangential component of $\mathbf{B}$ over this surface. In the simplified (scalar) version the quantity minimized is instead the variance of the scalar potential over the source surface. The scalar formulation greatly reduces the time required to compute required matrix elements, while imposing essentially the same physical boundary condition as the vector formulation (viz., that the coronal magnetic field be, as nearly as possible, normal to the source surface for continuity with the heliosphere). The source surface proposed for actual application is a surface of constant $\tilde{F} \equiv r^{-k} \tilde{B}$, where $r$ is the heliocentric distance and $\tilde{B}$ is the scalar magnitude of the $\mathbf{B}$ field produced by currents inside the Sun. Comparison with MHD simulations suggests that $k \approx 1.4$ is a good choice for the adjustable exponent. This value has been shown to map the neutral line on the source surface during Carrington Rotation 1869 (May-June 1993) to a range of latitudes that would have just grazed the position of Ulysses during that month in which sector structure disappeared from Ulysses' magnetometer observations.
\end{abstract}

\section{Background}

The magnetic field in the solar corona is traditionally modeled (Schatten et al., 1969; Altschuler and Newkirk, 1969; Hoeksema and Scherrer, 1986; Hoeksema, 1991) as being current-free (and thus derivable from a scalar potential) within a spherically annular volume of inner radius $1 r_{\odot}$ and outer radius $r_{0} \approx 1.6-2.5 r_{\odot}$ surrounding the Sun. The inner sphere $\left(r=r_{\odot}\right)$ corresponds to the photosphere, at which the line-of-sight component of the Sun's magnetic field B is remotely observed by means of Earth-based Zeeman spectroscopy. The outer sphere $\left(r=r_{0}\right)$ is regarded as a magnetic equipotential surface, to which $\mathbf{B}$ is therefore perpendicular and from which the solar wind implicitly flows radially outward into the heliosphere. Schatten et al. (1969) chose $r_{0}=1.6 r_{\odot}$ for the radius of their source surface. Altschuler and Newkirk (1969) chose $r_{0}=2.5 r_{\odot}$ in order to achieve better agreement with dimensions of large coronal helmet structures seen in eclipse photographs.

Subsequent magnetohydrodynamic (MHD) simulations by Pneuman and Kopp (1971a, b) showed (as many eclipse photographs had already indicated) that the outflow of solar wind must be significantly nonradial in the inner heliosphere. The dashed curves in Fig. 1a represent field lines obtained from the MHD simulation. The solid curves in Fig. 1a represent field lines obtained from a dipole centered within a spherical source surface of radius $r=2.5 r_{\odot}$. The disagreement at $r \gtrsim 2 r_{\odot}$ is quite substantial. These considerations have prompted various alternative formulations of the source-surface model. In the best-known of these alternatives, Schatten (1971) distinguished between a source surface at $r=1.6 r_{\odot}$ and a zero-potential surface at $r=2.5 r_{\odot}$. He actually used the solution from $r_{0}=2.5 r_{\odot}$ only at $r \leq 1.6 r_{\odot}$ and solved a second (exterior) potential problem to obtain $\mathbf{B}$ at $r \geq 1.6 r_{\odot}$ from the absolute value of $B_{r}$ at $r=1.6 r_{\odot}$. Zhao and 
Hoeksema (1995) currently use a modified version of Schatten's approach, treating these two radii as adjustable parameters of their model (see also Wang and Sheeley, 1995).

In a lesser known alternative formulation, Schulz et al. (1978) gave the source surface of Altschuler and Newkirk (1969) a non-spherical shape (still enclosing a volume $\approx 125 \pi r_{\odot}^{3} / 6$ ) but preserved its role as a magnetic equipotential surface from which the solar wind flows normally outward along straight lines. The particular shape of source surface chosen by Schulz et al. (1978) to illustrate the new model was an isogauss of the Sun's dipole field. The equation of this source surface (of equatorial radius $\left.r_{0} \approx 2.3 r_{\odot}\right)$ was $r=\left(1+3 \cos ^{2} \theta\right)^{1 / 6} r_{0}$, where $\theta$ denotes the magnetic colatitude. The essential feature of this surface was its prolateness along the dipole axis.

Schulz et al. (1978) found that rectilinear trajectories extending normally outward from such a source surface adequately simulated the field-line directions obtained by Pneuman and Kopp (1971a, b) from their MHD solution (see Fig. 1b) and adequately spread magnetic field lines throughout the heliosphere, so that the meridional $(\approx$ radial) component of $\mathbf{B}$ was virtually independent of magnetic latitude at any specified $r \gtrsim 1$ AU. Ulysses observations by Smith and Balogh (1995) have confirmed this (the latitude-independence of $r^{2} B_{r}$ ) as a desirable result.

Levine et al. (1982) applied the concept of Schulz et al. (1978) to a more realistic (not purely dipolar) heliomagnetic field. The magnetic field $\mathbf{B}=\tilde{\mathbf{B}}+\overline{\mathbf{B}}$ in the region $r_{\odot} \leq r \leq r_{s}(\theta, \varphi)$ between the photosphere and the non-spherical source surface was again separated into contributions due to currents at $r<r_{\odot}$ and at $r \geq r_{s}(\theta, \varphi)$, respectively, so that

$$
\tilde{\mathbf{B}}=-r_{\odot} \nabla \sum_{n=1}^{N} \sum_{m=0}^{n}\left(r_{\odot} / r\right)^{n+1}\left[g_{n}^{m} \cos m \varphi+h_{n}^{m} \sin m \varphi\right] \mathrm{P}_{n}^{m}(\theta)
$$

$$
\begin{array}{r}
\overline{\mathbf{B}}=-\left(r_{\odot}^{3} / r_{0}^{2}\right) \nabla \sum_{n=1}^{\bar{N}} \sum_{m=0}^{n}\left(r / r_{0}\right)^{n}\left[\bar{g}_{n}^{m} \cos m \varphi\right. \\
\left.+\bar{h}_{n}^{m} \sin m \varphi\right] \mathrm{P}_{n}^{m}(\theta)
\end{array}
$$

where $r_{0}$ is a nominal (e.g., equatorial) radius characterizing the source surface and thus normalizing the expansion coefficients $\left\{\bar{g}_{n}^{m}, \bar{h}_{n}^{m}\right\}$ in Eq. (1b).

The expansion coefficients $\left\{\bar{g}_{n}^{m}, \bar{h}_{n}^{m}\right\}$ in the work of Levine et al. (1982) were obtained by minimizing (with respect to the $\left\{\bar{g}_{n}^{m}, \bar{h}_{n}^{m}\right\}$ ) a bilinear variational quantity equal to the mean-square tangential component of $\mathbf{B}$ over the source surface. (The goal of this procedure was to make $\mathbf{B}$ as nearly as possible normal to the source surface from the inside.) The value of $r=r_{\mathrm{s}}(\theta, \varphi)$ on the source surface was implicitly specified by adopting a compromise between a source surface of constant $\tilde{B}^{2}$ (as in Schulz et al., 1978) and a source surface of constant $r$ (as in Altschuler and Newkirk, 1969). The present work offers a simplified prescription of the source surface and a streamlined determination of the coefficients $\left\{\bar{g}_{n}^{m}, \bar{h}_{n}^{m}\right\}$, in the hope that these steps will make the model easier to use in practice.

\section{Source surface}

The source surface in the present work is regarded (Schulz, 1995) as a surface of constant $\tilde{F} \equiv r^{-k} \tilde{B}$, where $\tilde{B}(\equiv|\tilde{\mathbf{B}}|)$ is the absolute value of the magnetic field $\tilde{\mathbf{B}}$ produced by currents inside the Sun and $k(\geq 0)$ is an
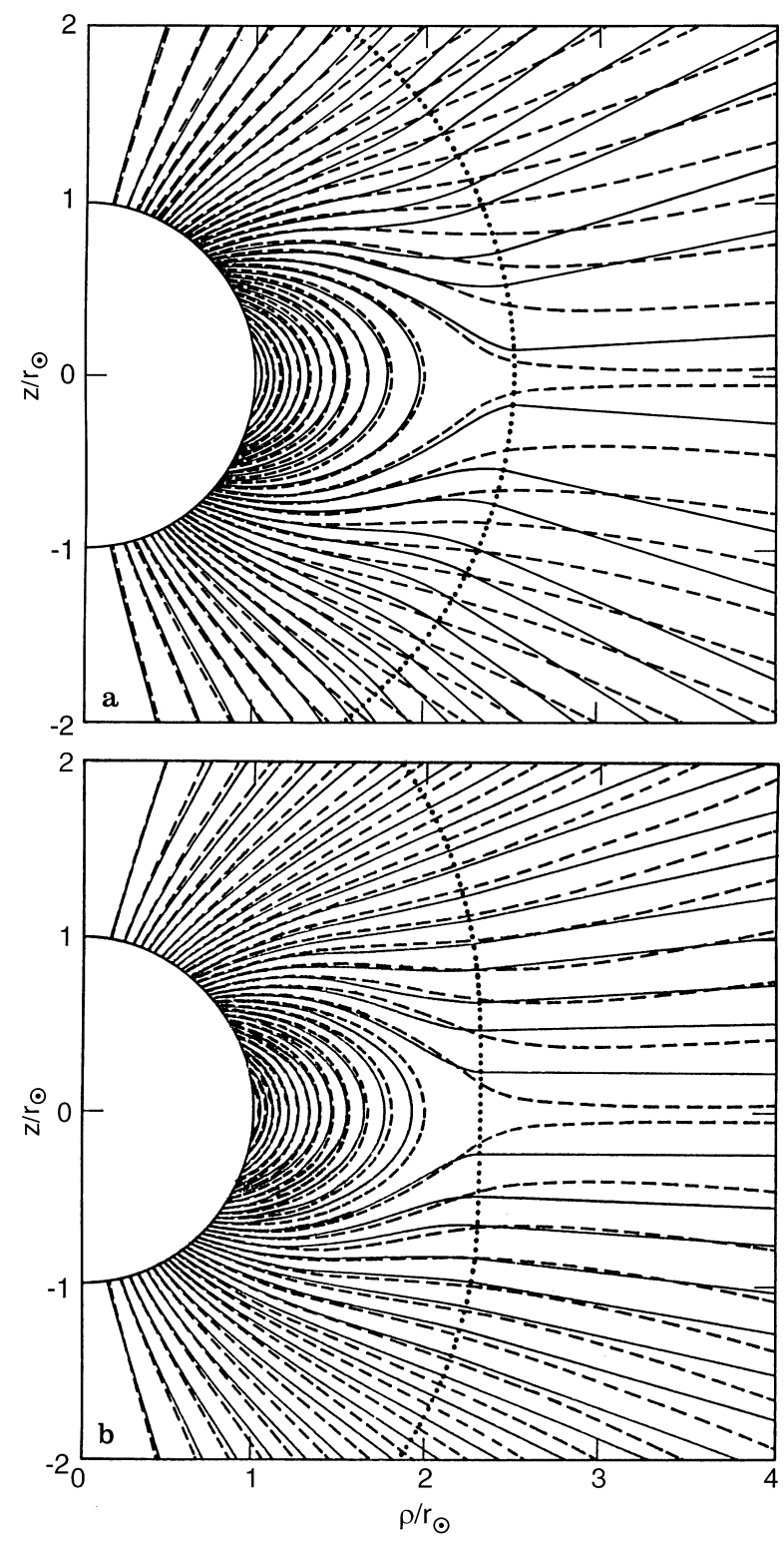

Fig. 1a,b. Representative field lines emanating from selected photospheric latitudes in various coronal magnetic-field models, applied to the case in which the Sun's field is dipolar. Dashed curves correspond to MHD model of Pneuman and Kopp (1971a,b) in both panels. Solid curves correspond to source-surface models: a with spherical source surface (dotted curve) of radius $r=2.5 r_{\odot}$ (Altschuler and Newkirk, 1969), corresponding to $k=\infty$ in present notation; and b with "dipole-isogauss" source surface (dotted curve) of variable radius $r=2.3\left(1+3 \cos ^{2}\right)^{1 / 6} r_{\odot}$ (Schulz et al., 1978), corresponding to $k=0$ in present notation $(\theta \equiv$ magnetic colatitude). 
adjustable parameter. The limit $k \rightarrow \infty$ leads back to the usual spherical source surface (Schatten et al., 1969; Altschuler and Newkirk, 1969), which was intended primarily for modeling the corona (not the entire heliosphere). The choice $k=0$ leads back to a source surface of constant $\tilde{B}$ (Schulz et al., 1978). For the special (test) case of a dipolar $\tilde{\mathbf{B}}$ field (but for general $k$ ) the equation of a source surface with equatorial radius $r_{0}$ would be

$r=\left(1+3 \cos ^{2} \theta\right)^{1 /(2 k+6)} r_{0}$,

where $\theta$ is the magnetic colatitude. The unit vector $\hat{\mathbf{n}}$ normal to the source surface would be given in spherical coordinates by

$$
\begin{aligned}
\hat{\mathbf{n}} & \equiv-|\nabla \tilde{F}|^{-1} \nabla \tilde{F} \\
& =\frac{3 \hat{\theta} \cos \theta \sin \theta+(k+3) \hat{\mathbf{r}}\left(1+3 \cos ^{2} \theta\right)}{\left[9 \cos ^{2} \theta \sin ^{2} \theta+(k+3)^{2}\left(1+3 \cos ^{2} \theta\right)^{2}\right]^{1 / 2}} .
\end{aligned}
$$

The direction of $\hat{\mathbf{n}}$ is easier to visualize in cylindrical coordinates $(\rho \equiv r \sin \theta, z \equiv r \cos \theta)$, since the value of

$$
\frac{\mathrm{d} z}{\mathrm{~d} \rho}=\frac{\left[k+3(k+4) \cos ^{2} \theta\right] \cos \theta}{\left[(k+3)+3(k+4) \cos ^{2} \theta\right] \sin \theta}
$$

along $\hat{\mathbf{n}}$ at the source surface is equal to the tangent of the heliomagnetic latitude $\lambda$ to which the corresponding field line would map asymptotically. This $\mathrm{d} z / \mathrm{d} \rho$ should well approximate the value of $\tan \lambda$ along the field line at $r \sim 1.1-5.4$ AU (the range spanned by Ulysses). However, the actual equation of a solar-wind streamline, from the source surface to the boundary of the heliosphere in this model, is

$z=z_{\mathrm{S}}+(\mathrm{d} z / \mathrm{d} \rho)_{\mathrm{s}}\left(\rho-\rho_{\mathrm{s}}\right)$,

where the subscript $\mathrm{s}$ denotes evaluation at the source surface (Schulz et al., 1978).

The dotted curve in Fig. 2 corresponds to the source surface specified by Eq. (2) for $k=1.4$, with $r_{0}$ chosen so as to preserve the enclosed volume $\left(=125 \pi r_{\odot}^{3} / 6\right)$. In this case a source surface of equatorial radius $r_{0} \approx 2.33 r_{\odot}$ would enclose the same volume as a sphere of radius $2.5 r_{\odot}$. The solid lines in Fig. 2 extend normally outward from selected latitudes ( $5^{\circ}$ apart) on the source surface, for comparison with the directions of MHD field lines (dashed curves) obtained by Pneuman and Kopp (1971a, b). This comparison confirms that $k \approx 1.4$ is a reasonable choice for the shape parameter $k$ in Eq. (2), at least for the dipolar test case. Moreover, the choice of $k=1.4$ has been found (Schulz, 1995) to account within $1^{\circ}$ for the maximum latitude $\left(\approx 30^{\circ} \mathrm{S}\right)$ reached by the heliospheric current sheet (HCS) at $r \approx 4.7$ AU during May 1993, when "sector structure" first disappeared from the Ulysses magnetometer observations (Smith et al., 1993).

Since the Sun's B field is predominantly dipolar only during part of the solar cycle, extending for a few years on either side of solar minimum, it may be premature to infer that the same choice for $k$ should apply under solar-maximum conditions also. For now this is just a working hypothesis to be tested against future Ulysses data. However, Levine et al. (1982) found that a similarly (although parametrically more cumbersome) intermediate shape between $k=0$ and $k=\infty$ could account quite well not only for the MHD field configuration obtained by Pneuman and Kopp (1971a,b) in the dipolar test case, but also for a more complicated coronal and interplanetary B-field configuration encountered not far from solar maximum.

\section{Variational principle}

The magnetic field $\mathbf{B}(=\tilde{\mathbf{B}}+\overline{\mathbf{B}})$ specified by Eq. (1) is derivable from a scalar potential $V(=\tilde{V}+\bar{V})$ at $r \leq r_{0}$. The vector $\mathbf{B}$ is made everywhere perpendicular to the spherical source surface (of radius $r_{0}$ ) employed in the model of Schatten et al. (1969) or Altschuler and Newkirk (1969) by choosing the expansion coefficients

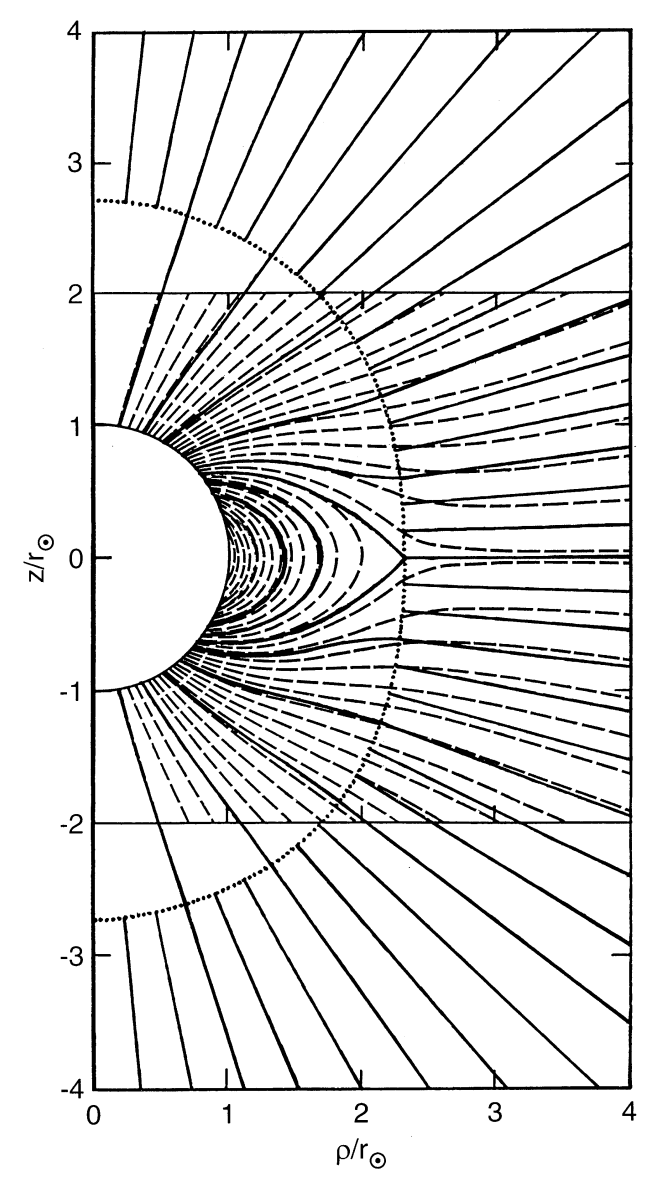

Fig. 2. Dashed curves represent field lines emanating from selected photospheric latitudes in MHD model of Pneuman and Kopp (1971a, b) for the case in which the Sun's field is dipolar. Solid curves represent field lines that cross the source surface, specified as $r=2.33\left(1+3 \cos ^{2} \theta\right)^{5 / 44} r_{\odot}$, at $15^{\circ}$ intervals of magnetic latitude in the present model $(k=7 / 5)$. This value of $k$ best reproduces the latitude-dependence of $\mathbf{B}$-field direction in the inner heliosphere (just outside the source surface, where field lines are straight lines in the present model). Portions of additional field lines in the present model are shown as solid lines extending outward from the source surface at $5^{\circ}$ intervals of magnetic latitude. These are truncated at the source surface in order not to clutter the presentation. 
$\left\{\bar{g}_{n}^{m}, \bar{h}_{n}^{m}\right\}$ in Eq. $(1 \mathrm{~b})$ so that $\left(\bar{g}_{n}^{m}, \bar{h}_{n}^{m}\right)=\left(g_{n}^{m}, h_{n}^{m}\right)$. This choice of $\left\{\bar{g}_{n}^{m}, \bar{h}_{n}^{m}\right\}$ makes the source surface $\left(r=r_{0}\right)$ an equipotential of $V$. Indeed, it makes $V=0$ (regardless of $\theta$ and $\varphi$ ) at $r=r_{0}$.

The magnetic field $\mathbf{B}=-\nabla V$ specified by Eq. (1) is made (as nearly as possible) perpendicular to a prolate source surface in the model of Schulz et al. (1978) and Levine et al. (1982) by minimizing the variational quantity

$\sigma \equiv \oint_{\mathrm{ss}}(\mathbf{n} \times \mathbf{B})^{2} \mathrm{~d} A$

with respect to the expansion coefficients $\left\{\bar{g}_{n}^{m},,_{n}^{m}\right\}$ in Eq. (1b). This procedure seems simple enough in concept, but its implementation in general has led to page-long equations and correspondingly long computation times (Levine et al., 1982).

The present work offers a simpler procedure for making B (as nearly as possible) normal to the source surface from the inside. The variational quantity minimized here is

$\sigma^{*} \equiv \oint_{\text {ss }}(V-\langle V\rangle)^{2} \mathrm{~d} A$

where $\langle V\rangle$ denotes the mean value of $V$ over the source surface. (It is easy to show that, if the goal is to minimize the mean-square deviation of $V$ from a constant over the source surface, then that constant must be $\langle V\rangle$ ). As long as $\mathbf{B}$ is to be derived from a scalar potential in the region surrounded by the source surface, a scalar formulation based on Eq. (7) seems simpler to implement than a vector formulation based on Eq. (6). The results should be equivalent, if not identical.

The coefficients $\left\{g_{n}^{m}, h_{n}^{m}\right\}$ are presumed to have been determined (e.g., Hoeksema and Scherrer, 1986) from the line-of-sight component of $\mathbf{B}$, as measured through Earth-based Zeeman spectroscopy. That deconvolution typically invokes a spherical source surface of radius $r=2.5 r_{\odot}$. Better values for the coefficients $\left\{g_{n}^{m}, h_{n}^{m}\right\}$ could possibly be deduced through use of a prolate source surface, but this potential refinement (which would seem to require an iterative deconvolution of the solar magnetic-field data) is not addressed here. Only the coefficients $\left\{\bar{g}_{n}^{m}, \bar{h}_{n}^{m}\right\}$ are evaluated via Eq. (7) in the present work.

Since $\sigma^{*}$ is algebraically bilinear in the expansion coefficients $\left\{\bar{g}_{n}^{m}, \bar{h}_{n}^{m}\right\}$, the equations $\mathrm{d} \sigma^{*} / \mathrm{d} \bar{g}_{n^{\prime}}^{m^{\prime}}=\mathrm{d} \sigma^{*} / \mathrm{d} \bar{h}_{n^{\prime}}^{m^{\prime}}$ $=0$ suffice to determine optimal values for the $\bar{N}(\bar{N}+2)$ coefficients $\left\{\bar{g}_{n}^{m}, \bar{h}_{n}^{m}\right\}$. There are $(\bar{N} / 2)(\bar{N}+3)$ linear equations of the form

$$
\begin{aligned}
\frac{\partial \sigma^{*}}{\partial \bar{g}_{n^{\prime}}^{m^{\prime}}} & =2 \oint_{\text {ss }}(V-\langle V\rangle)\left[\frac{\partial V}{\partial \bar{g}_{n^{\prime}}^{m^{\prime}}}-\frac{\partial\langle V\rangle}{\partial \bar{g}_{n^{\prime}}^{m^{\prime}}}\right] \mathrm{d} A \\
& =2 \oint_{\text {ss }}(V-\langle V\rangle)\left[\partial V / \bar{g}_{n^{\prime}}^{m^{\prime}}\right] \mathrm{d} A=0,
\end{aligned}
$$

corresponding to distinct values of $\left(n^{\prime}, m^{\prime}\right)$, and $(\bar{N} / 2)(\bar{N}+1)$ linear equations of the complementary form

$$
\begin{aligned}
\frac{\partial \sigma^{*}}{\partial \bar{h}_{n^{\prime}}^{m^{\prime}}} & =2 \oint_{\mathrm{ss}}(V-\langle V\rangle)\left[\frac{\partial V}{\partial \bar{h}_{n^{\prime}}^{m^{\prime}}}-\frac{\partial\langle V\rangle}{\partial \bar{h}_{n^{\prime}}^{m^{\prime}}}\right] \mathrm{d} A \\
& =2 \oint_{\mathrm{ss}}(V-\langle V\rangle)\left[\partial V / \partial \bar{h}_{n^{\prime}}^{m^{\prime}}\right] \mathrm{d} A=0,
\end{aligned}
$$

also corresponding to distinct values of $\left(n^{\prime}, m^{\prime}\right)$. The parenthesized factor in Eq. (8) is linear with respect to the $\left\{\bar{g}_{n}^{m}, \bar{h}_{n}^{m}\right\}$ in each case; the factors in square brackets can depend on $\left(n^{\prime}, m^{\prime}\right)$ but not explicitly on $\left\{\bar{g}_{n^{\prime}}^{m^{\prime}}, \bar{h}_{n^{\prime}}^{m^{\prime}}\right\}$, since $V$ itself depends linearly on $\left\{\bar{g}_{n^{\prime}}^{m^{\prime}}, \bar{h}_{n^{\prime}}^{m^{\prime}}\right\}$ in that factor. The terms $\partial\langle V\rangle / \partial \bar{g}_{n^{\prime}}^{m^{\prime}}$ and $\partial\langle V\rangle / \partial \bar{h}_{n^{\prime}}^{m^{\prime}}$ can be removed from the square-bracketed factors in Eqs. (8a) and (8b), respectively, because they are independent of spatial coordinates. Thus, they behave as constants in any integrations over the source surface of whatever shape, which thus reduce to integrals of $(V-\langle V\rangle)$ with respect to $\mathrm{d} A$ over the source surface. Such integrals vanish by definition: $\langle V\rangle$ is the mean value of $V$ over the source surface. A further simplification (such that $\langle V\rangle=0$ ) occurs if $\tilde{\mathbf{B}}$ (and thus the source surface itself) is adequately symmetric between north and south. Otherwise it is necessary to implement Eqs. (8a) and (8b) as they stand, accepting that $\langle V\rangle$ can depend on $\left\{\bar{g}_{n}^{m^{\prime}}, \bar{h}_{n}^{m^{\prime}}\right\}$ if $\langle V\rangle \not \equiv 0$.

\section{Test case}

The greatly simplified case of dipolar $\tilde{\mathbf{B}}$ illustrates how this described variational procedure might work in practice. The source surface in this case reduces to that specified by Eq. (2), and the magnetic field $\mathbf{B}$ given by Eq. (1) becomes derivable from the scalar potential

$$
\begin{aligned}
V(r, \theta)= & g_{1}^{0}\left(r_{\odot}^{3} / r^{2}\right) \cos \theta \\
& +\left(r_{\odot}^{3} / r_{0}^{2}\right) \sum_{n=1}^{\bar{N}}\left(r / r_{0}\right)^{n} \bar{g}_{n}^{0} \mathrm{P}_{n}(\cos \theta),
\end{aligned}
$$

with only odd values of $n(=1,3,5, \ldots)$ represented in the summation. Legendre functions

$\mathrm{P}_{n}(x)=\frac{1}{n ! 2^{n}} \frac{\mathrm{d}^{n}}{\mathrm{~d} x^{n}}\left[\left(x^{2}-1\right)^{n}\right]$

satisfy the differential equation

$\frac{\mathrm{d}}{\mathrm{d} x}\left[\left(1-x^{2}\right) \frac{\mathrm{dP}_{n}}{\mathrm{~d} x}\right]+n(n+1) \mathrm{P}_{n}(x)=0$.

It follows from Eq. (9) that $\mathrm{d} V / \mathrm{d} \bar{g}_{n^{\prime}}^{0}=\left(r_{\odot}^{3} / r_{0}^{2}\right) \times$ $\left(r / r_{0}\right)^{n^{\prime}} \mathrm{P}_{n^{\prime}}(x)$, where $x=\cos \theta \equiv \mathrm{P}_{1}(x)$. Thus, it follows from Eq. (8a) that

$$
\begin{aligned}
\sum_{n=1}^{\bar{N}} \bar{g}_{n}^{0} \oint_{\mathrm{ss}}\left(r / r_{0}\right)^{n+n^{\prime}} \mathbf{P}_{n}(x) \mathbf{P}_{n^{\prime}}(x) \mathrm{d} A \\
=-g_{1}^{0} \oint_{\mathrm{ss}}\left(r / r_{0}\right)^{n^{\prime}-2} x \mathrm{P}_{n^{\prime}}(x) \mathrm{d} A
\end{aligned}
$$

with $r / r_{0}$ as given by Eq. (2) and 
Table 1. Optimized values of $\bar{g}_{n}^{0} / g_{1}^{0}$ for selected values of $\bar{N}$

\begin{tabular}{rlllllll}
\hline$n$ & $\bar{N}=3$ & $\bar{N}=5$ & $\bar{N}=7$ & $\bar{N}=9$ & $\bar{N}=11$ & $\bar{N}=13$ & $\bar{N}=15$ \\
\hline 1 & -0.723974 & -0.725645 & -0.725908 & -0.725957 & -0.725967 & -0.725970 & -0.725970 \\
3 & +0.0941047 & +0.103736 & +0.105261 & +0.105550 & +0.105610 & +0.105624 & +0.105627 \\
5 & 0.0 & -0.0274149 & -0.0324136 & -0.0334301 & -0.0336532 & -0.0337049 & -0.0337174 \\
7 & 0.0 & 0.0 & +0.00944986 & +0.0119309 & +0.0125432 & +0.0126956 & +0.0127342 \\
9 & 0.0 & 0.0 & 0.0 & -0.00351930 & -0.00472703 & -0.00507664 & -0.00517369 \\
11 & 0.0 & 0.0 & 0.0 & 0.0 & +0.00137132 & +0.00195296 & +0.00214590 \\
12 & 0.0 & 0.0 & 0.0 & 0.0 & 0.0 & -0.000550607 & -0.000828866 \\
13 & 0.0 & 0.0 & 0.0 & 0.0 & 0.0 & 0.0 & +0.000225862 \\
\hline
\end{tabular}

$$
\mathrm{d} A=4 \pi r^{2}\left[1+\frac{3 x^{2}\left(1-x^{2}\right)}{(k+3)^{2}\left(1+3 x^{2}\right)^{2}}\right]^{1 / 2} \mathrm{~d} x
$$

for $0 \leq x \leq 1$. The required integrals have been evaluated numerically with Mathematica (Wolfram, 1992), which has also been programmed to solve the resulting simultaneous linear equations specified by Eq. (11). Results for $\left\{\bar{g}_{n}^{0} / g_{1}^{0}\right\}$ corresponding to selected values of $\bar{N}(=3,5,7, \ldots, 15)$ are listed in Table 1 . Truncation of Eq. (1b) at $=1$ would be contrary to the spirit of the model but is found to yield $\bar{g}_{n}^{0} / g_{1}^{0}=-0.708580$.

The values of $\bar{g}_{n}^{0} / g_{1}^{0}$ in Table 1 represent normalized $2^{n}$-pole moments due to azimuthal currents that flow outside the source surface. These values specify the relative weights that should be assigned to the various $\mathrm{P}_{n}^{0}(\theta)$ in Eq. (1b) if the Sun's own magnetic field ( $\left.\tilde{\mathbf{B}}\right)$ were purely dipolar. The value of $\bar{g}_{n}^{0} / g_{1}^{0}$ in any row of Table 1 seems to approach a limit as $\bar{N} \rightarrow \infty$, but the values of $\left\{\bar{g}_{n}^{0} / g_{1}^{0}\right\}$ in any column of Table 1 are mutually optimized for the corresponding $\bar{N}$. Strictly speaking, this means that Eq. (1b) is not a uniformly convergent representation of $\overline{\mathbf{B}}$ unless $k=\infty$ (i.e., unless the source surface is spherical). One cannot just truncate any column in Table 1 at some $n<\bar{N}$ and expect the resulting representation to be "good enough." Even so, there is little visible difference between the $\bar{N}=13$ model and the $\bar{N}=11$ or the $\bar{N}=15$ model when representative field lines are plotted for comparison with Pneuman and Kopp (1971a, b).

Magnetic field lines in heliospheric models are usually traced numerically (e.g., by using a fourth-order RungeKutta technique) as space curves locally tangential to $\mathbf{B}$. However, the tracing procedure can be greatly simplified, at least for an axisymmetric $\mathbf{B}$ field derived from a scalar potential, by formally constructing Euler potentials $(\alpha, \beta)$ such that $\beta=\varphi$ and $\mathbf{B}=\nabla \alpha \times \nabla \beta=-\nabla V$ (e.g., Stern, 1976, 1994)

$\frac{1}{r \sin \theta}\left[\frac{\hat{\mathbf{r}}}{r} \frac{\partial \alpha}{\partial \theta}-\hat{\theta} \frac{\partial \alpha}{\partial r}\right]=\hat{\mathbf{r}} \frac{\partial V}{\partial r}+\frac{\hat{\theta}}{r} \frac{\partial V}{\partial \theta}$

This means that

$\partial \alpha / \partial \theta=-r^{2} \sin \theta(\partial V / \partial r)$

and that

$\partial \alpha / \partial r=\sin \theta(\partial V / \partial \theta)$ for the case of a current-free axisymmetric $\mathbf{B}$ field. (This is admittedly a very special test case, unlikely to be encountered in nature.) Thus, it follows from Eq. (13a) that

$$
\begin{aligned}
\alpha(r, \theta) & =g_{1}^{0}\left(r_{\odot}^{3} / r\right)\left(1-x^{2}\right) \\
& +\left(r_{\odot}^{3} / r_{0}\right) \sum_{n=1}^{\bar{N}} \frac{\left(r / r_{0}\right)^{n+1} \bar{g}_{n}^{0}}{(n-1) ! 2^{n}} \frac{\mathrm{d}^{n-1}}{\mathrm{~d} x^{n-1}}\left[\left(x^{2}-1\right)^{n}\right]
\end{aligned}
$$

and from Eq. (13b) that

$$
\begin{aligned}
\alpha(r, \theta)= & g_{1}^{0}\left(r_{\odot}^{3} / r\right)\left(1-x^{2}\right) \\
& -\left(r_{\odot}^{3} / r_{0}\right)\left(1-x^{2}\right) \sum_{n=1}^{\bar{N}} \frac{\left(r / r_{0}\right)^{n+1} \bar{g}_{n}^{0}}{(n+1) ! 2^{n}} \frac{\mathrm{d}^{n+1}}{\mathrm{~d} x^{n+1}} \\
& {\left[\left(x^{2}-1\right)^{n}\right] . }
\end{aligned}
$$

That these two expressions for $\alpha(r, \theta)$ are equivalent can be seen by integrating Eq. (10b) with respect to $x$. The latter form of Eq. (14) is probably the more convenient, however: This form shows $\sin ^{2} \theta\left(=1-x^{2}\right)$ explicitly as a factor common to all terms in the summation, leaving the derivative (rather than the integral) of $\mathrm{P}_{n}(x)$ with respect to $x$ to be generated (as a callable function) by Mathematica (Wolfram, 1992).

The value of $\alpha$ at any point along a field line must be equal to $1 / 2 \pi$ times the magnetic flux enclosed by rotating that field line about the symmetry axis. That $\alpha$ and $\beta$ remain constant along any field line is well known and obvious, since $\nabla \alpha \cdot(\nabla \alpha \times \nabla \beta)=\nabla \beta \cdot(\nabla \alpha \times \nabla \beta)$ $=0$. (The cross product between any two vectors is always perpendicular to each.) Thus, it follows from Eq. (14b) that the quantity

$$
\frac{1}{L}=\left(1-x^{2}\right)\left(r_{\odot} / r\right)\left[1-\sum_{n=1}^{\bar{N}} \frac{\left(r / r_{0}\right)^{n+2}\left(\bar{g}_{n}^{0} / g_{1}^{0}\right)}{(n+1)} \frac{\mathrm{dP}_{n}}{\mathrm{~d} x}\right]
$$

should remain constant on the magnetic surface (shell of field lines) that it labels. (The " $L$ " terminology is borrowed from magnetospheric physics. The idea is that a dipolar field line would satisfy the equation $r=L r_{\odot} \sin ^{2} \theta$ and so would intersect the photosphere at a magnetic latitude $\Lambda$ such that $\cos \Lambda=L^{-1 / 2}$.) In a field geometry stretched by the heliospheric current sheet, the enclosed magnetic flux remains proportional to $1 / L$, but the field line intersects the equator at $r>L r_{\odot}$ and the photosphere at a magnetic latitude slightly higher than $\cos ^{-1}\left(L^{-1 / 2}\right)$. Thus, for example, the last 
closed field line (which reaches $x \equiv \cos \theta=0$ at $\left.r=r_{0}=2.33 r_{\odot}\right)$ in the present model bears the label $L^{*}=1.6425$ and crosses the photosphere at magnetic latitude $\Lambda^{*}=39.69^{\circ}$. A purely dipolar field line with $L=1.6425$ would cross the photosphere at $\Lambda=38.71^{\circ}$. For a spherical source surface of radius $r=2.5 r_{\odot}$, the last closed field line would have borne the label $L^{*}=5 / 3$ and would have intersected the photosphere at $\Lambda^{*}=40.32^{\circ}$. These differences are not major. Moreover, the present source-surface model (with $k=1.4$ ) and the model with a spherical source surface $(k=\infty)$ differ only slightly with respect to the field-line configuration at $r \lesssim 0.8 r_{0}$. Decisive differences in field-line configuration appear only in the outer part of the region enclosed by the source surface and (especially) in the mapping of field lines from the source surface into the heliosphere.

The solid curves in Fig. 2 are selected field lines constructed via Eq. (15) from the values of $\bar{g}_{n}^{0} / g_{1}^{0}$ shown in Table 1 for $\bar{N}=15$. These field lines have been chosen so as to intersect the source surface at $15^{\circ}$ intervals of magnetic latitude. The superposed dashed curves are field lines from the MHD simulation of Pneuman and Kopp (1971a, b), as in Fig. 1. These had been chosen so as to intersect the photosphere at a convenient set of latitudes (not evenly spaced). Thus, the dashed and solid curves in Fig. 2 do not correspond one-to-one. The noteworthy feature is their "nested" relationship: They tend to interleave rather than intersect.

\section{Heliospheric field intensity}

The amount of magnetic flux poleward of the magnetic shell that bears the label $\alpha\left(=g_{1}^{0} r_{\odot}^{2} / L\right)$ is equal to $2 \pi \alpha$, and so the mean value of $\left|B_{r}\right|$ over any heliocentric sphere of radius $r \geq r_{0}$ must be equal to $\left|g_{1}^{0} r_{\odot}^{2} / r^{2} L^{*}\right|$. Since $L^{*}=1.6425$ (see earlier), this means that $\left\langle(r / \mathrm{AU})^{2}\left|B_{r}\right|\right\rangle \approx 1.317 \times 10^{-5}\left|g_{1}^{0}\right|$ if $r / \mathrm{AU}$ is the heliocentric distance measured in $\mathrm{AU}\left(1 \mathrm{AU} \approx 215 r_{\odot}\right)$. The latitudinal distribution of this field intensity is of interest here for comparison with Ulysses results.

Since the construction of field lines outside the source surface is essentially geometrical, it is easy to calculate the corresponding field intensity geometrically (by invoking magnetic flux conservation). The solid straight lines in Fig. 2 are normal to the source surface, and each can be considered to radiate from a center of curvature in the same meridional plane (Schulz et al., 1978, Fig. 4). The radius of curvature of a magnetic meridian on the source surface is given by

$$
B_{r}= \pm 0.135693 g_{1}^{0}\left[2.330329 r_{\odot} /\left(r-0.397215 r_{\odot}\right)\right]^{2}
$$

since the surrounding field lines would fill an infinitesimal cone with its apex at $z= \pm[3 /(4 k+15)] 2^{1 /(k+3)} r_{0}$ along the axis of symmetry (see Schulz et al., 1978).

The geometry is a bit more complicated for mappings from other latitudes on the source surface, but the principle is the same: The meridional component of $\mathbf{B}$ should vary as the ratio between corresponding infinitesimal areas (transverse to the solar-wind velocity) on the source surface and (as mapped to the point of interest) in the heliosphere. The ratio of such areas can be expressed (Schulz et al., 1978) as

$$
\begin{aligned}
\frac{B_{m}}{B_{\mathrm{s}}} & =\frac{r_{c} \rho_{s}}{\left\{r_{c}+\left[\left(\rho-\rho_{s}\right)^{2}+\left(z-z_{s}\right)^{2}\right]^{1 / 2}\right\} \rho} \\
& =\frac{r_{c} \rho_{s}}{\left\{r_{c}+\left(\rho-\rho_{s}\right)\left[1+(\mathrm{d} z / \mathrm{d} \rho)_{s}^{2}\right]^{1 / 2}\right\} \rho}
\end{aligned}
$$

where $(\rho, z)$ are the cylindrical coordinates of the point of interest in the heliosphere and $\left(\rho_{s}, z_{s}\right)$ are the cylindrical coordinates of the corresponding point on the source surface. The linear mapping of a field line from $\left(\rho_{s}, z_{s}\right)$ to $(\rho, z)$ is specified by Eq. (5). The meridional component of $\mathbf{B}$ (called $B_{m}$ in the heliosphere and $B_{s}$ on the source surface) is simultaneously equal to $\left(B_{r}^{2}+B_{\theta}^{2}\right)^{1 / 2}$ and $\left(B_{\rho}^{2}+B_{z}^{2}\right)^{1 / 2}$. This differs only very slightly from $B_{r}$, however, at heliocentric distances $r \gtrsim 1 \mathrm{AU}\left(\gg r_{c}\right)$.

It seems most convenient to map from a selected point $\left(\rho_{s}, z_{s}\right)$ on the source surface to a chosen heliocentric distance $r$ in the heliosphere. The corresponding value of $\left(\rho-\rho_{s}\right)$, which is needed in Eq. (18), can be obtained by solving the quadratic equation

$$
\begin{aligned}
r^{2}= & \left(\rho-\rho_{s}+\rho_{s}\right)^{2}+\left(z-z_{s}+z_{s}\right)^{2} \\
=[1 & \left.+(\mathrm{d} z / \mathrm{d} \rho)_{s}^{2}\right]\left(\rho-\rho_{s}\right)^{2}+r_{s}^{2} \\
& +2\left[\rho_{s}+z_{s}(\mathrm{~d} z / \mathrm{d} \rho)_{s}\right]\left(\rho-\rho_{s}\right)
\end{aligned}
$$

where $r_{s}^{2} \equiv \rho_{s}^{2}+z_{s}^{2}$. The solution for $\rho \geq \rho_{s}$ is

$$
\begin{aligned}
& {\left[1+(\mathrm{d} z / \mathrm{d} \rho)_{s}^{2}\right]\left(\rho-\rho_{s}\right)=} \\
& \left\{\left[\rho_{s}+z_{s}(\mathrm{~d} z / \mathrm{d} \rho)_{s}\right]^{2}+\left[1+(\mathrm{d} z / \mathrm{d} \rho)_{s}^{2}\right]\left(r^{2}-r_{s}^{2}\right)\right\}^{1 / 2} \\
& \quad-\left[\rho_{s}+z_{s}(\mathrm{~d} z / \mathrm{d} \rho)_{s}\right]
\end{aligned}
$$

The colatitude $\theta$ of the heliospheric point to which $\left(\rho_{s}, z_{s}\right)$ maps is indicated by Eq. (5). It is not practical to select the heliospheric point $(\rho, z)$ first and then map back to the source surface, since the value of $(d z / d \rho)_{s}$ needed for

$r_{c}=\frac{\left(1+3 \cos ^{2} \theta_{\mathrm{s}}\right)^{-(2 k+5) /(2 k+6)} r_{0}\left[\left(1+3 \cos ^{2} \theta_{\mathrm{s}}\right)^{2}(k+3)^{2}+9 \cos ^{2} \theta_{\mathrm{s}} \sin ^{2} \theta_{\mathrm{s}}\right]^{3 / 2}}{(k+3)\left[\left(1+3 \cos ^{2} \theta_{\mathrm{s}}\right)^{2}(k+3)^{2}+9 \cos ^{2} \theta_{\mathrm{s}} \sin ^{2} \theta_{\mathrm{s}}+3(k+3)\left(5 \cos ^{2} \theta_{\mathrm{s}}-1\right)\right]}$

which reduces to $r_{c}=(k+3)\left(r_{0} / k\right)=(22 / 7) r_{0}$ at the equator $\left(\theta_{s}=\pi / 2\right)$ and to $r_{c}=4[(k+3) /(4 k+15)] \times$ $2^{1 /(k+3)} r_{0}=1.000141 r_{0}$ at the poles $\left(\theta_{\mathrm{s}}=0, \pi\right)$ if $k=7 / 5$. The value of $B_{r}$ along either polar field line (beyond the source surface) thus varies as making this mapping is dependent on $\left(\rho_{s}, z_{S}\right)$ and would thus remain unknown until the mapping was completed.

The limit $r \rightarrow \infty$ yields $\left(\rho-\rho_{s}\right) \rho \rightarrow\left[1+(\mathrm{d} z / \mathrm{d} \rho)_{s}^{2}\right]^{-1} r^{2}$ and $\operatorname{ctn} \theta \rightarrow(\mathrm{d} z / \mathrm{d} \rho)_{s}$. The asymptotic value of $\left(r / r_{\odot}\right)^{2} B_{m}$ is thus given by 
$\left(r / r_{\odot}\right)^{2} B_{m} \rightarrow\left(r_{c} \rho_{s} / r_{\odot}^{2}\right)\left[1+(\mathrm{d} z / \mathrm{d} \rho)_{s}^{2}\right]^{1 / 2} B_{s}$.

This quantity is plotted (solid curve) in Fig. 3, but as a function of the heliomagnetic latitude $\lambda \equiv 90^{\circ}-\theta$. The latitude $\lambda$ along a field line specified by Eq. (5) approaches $\tan ^{-1}(\mathrm{~d} z / \mathrm{d} \rho)_{s}$ as $r \rightarrow \infty$. As would be expected, the asymptotic plot of $\left(r / r_{\odot}\right)^{2} B_{m}$ against $\lambda$ in Fig. 3 is almost indistinguishable from a plot of $\left(r / r_{\odot}\right)^{2} B_{m}$ against $\lambda$ at $r=1$ AU (not shown). The meridional (asymptotically radial) component of $\mathbf{B}$ in this model varies by about $\pm 18 \%$ from its mean value over about $80 \%$ of the unit sphere. A source-surface model with $k=1.4$ thus leads (not surprisingly) to a latitudinal distribution of magnetic flux similar to that found by Pneuman and Kopp (1971a, b).

The dashed step-function in Fig. 3 corresponds to a hypothetically uniform latitudinal distribution of the same amount of magnetic flux, which would be in better agreement with the Ulysses observations (Smith and Balogh, 1995). The meridional component of $\mathbf{B}$ at $r=1$ AU actually is almost latitude-independent (varying by less than $\pm 5 \%$ from its mean value over $85 \%$ of the unit sphere) in a source surface model with $k=0$ (Schulz et al., 1978), which corresponds to Fig. 1b here. A spherical source surface $(k=\infty$, corresponding to Fig. 1a here) would have led to a sinusoidal variation of $B_{r}$ with latitude: $B_{r}=3 g_{1}^{0}\left(r_{\odot}^{3} / r^{2} r_{0}\right) \sin \lambda$, which is contrary to all observations.

\section{Summary and discussion}

If the criterion for a successful heliospheric model were just that $\left(r / r_{\odot}\right)^{2} B_{m}$ be almost independent of $\lambda$ at $r \lesssim 1$ $\mathrm{AU}$, there might be reason to prefer the $k=0$ source

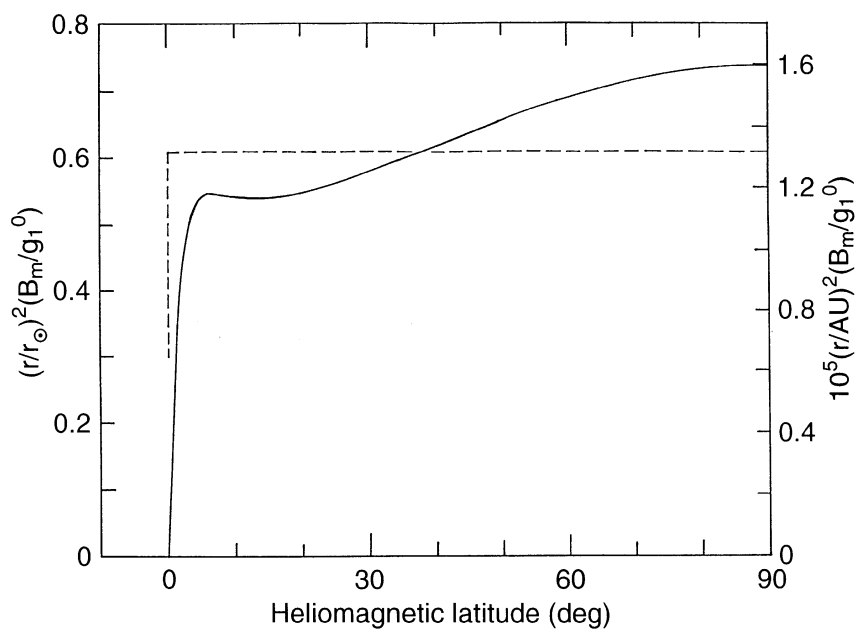

Fig. 3. Variation of meridional (asymptotically radial) component of $\left(r / r_{\odot}\right)^{2} \boldsymbol{B}$ [component denoted $\left(r / r_{\odot}\right)^{2} B_{m}$ ] with heliomagnetic latitude $\lambda\left(\equiv 90^{\circ}-\theta\right)$ in the limit $r \rightarrow \infty$, in a source-surface model with $k=7 / 5$ for the case in which the Sun's field is dipolar. For comparison (dashed step-function): hypothetical case (quite well approximated (Schulz et al., 1978) by a source-surface model with $k=0$ ) in which magnetic flux is uniformly distributed over either hemisphere surface (Schulz et al., 1978) over the present $k=1.4$ version. However, a comparison between Figs. 2 and $1 \mathrm{~b}$ confirms that the present version better accounts for the latitude-dependent direction of $\mathbf{B}$ outside the source surface, and Schulz (1995) found that the $k=1.4$ model accounts better (almost perfectly, in fact) for the Ulysses-inferred latitudinal extent (Smith et al., 1993) of the heliospheric current sheet (HCS) during Carrington Rotation 1869 (May-June 1993, when sector structure first disappeared from Ulysses' magnetometer observations).

It seems that only a slightly equatorward redistribution of the normal component of $\mathbf{B}$ over the present $(k=1.4)$ source surface could preserve the desired directionality and mapping properties achieved here, while also restoring the near-uniformity of $\left(r / r_{\odot}\right)^{2} B_{m}$ with magnetic latitude previously achieved with the $k=0$ model. Uniqueness of solutions to Laplace's equation for the Dirichlet problem (e.g., Jackson, 1962) posed here [and solved via Eq. (7)] precludes such a redistribution of $\hat{\mathbf{n}} \cdot \mathbf{B}$ within the framework of potential theory. Thus, further improvements in modeling the heliosphere by source-surface methods would seem to require that currents be permitted to flow inside the region surrounded by the source surface. This is not a radical requirement, of course: it seems quite reasonable that the corona would contain plasma currents that merge smoothly into the HCS. A source-surface model that precludes such currents is just too naive in the present context.

The dual source-surface model of Schatten (1971) already provides for a current-bearing coronal transition region $\left(1.6 r_{\odot} \lesssim r \lesssim 2.5 r_{\odot}\right)$ and thus partially answers the foregoing objection. Another interesting approach, however, would be to add currents implicitly through a B-field expansion not based on spherical harmonics (e.g., Schulz and Eviatar, 1969; Mead and Fairfield, 1975). This approach would, of course, require that Eq. (7) be abandoned in favor of Eq. (6), as a means of enforcing (in the least-squares sense) the usual boundary condition that calls for the tangential component of $\mathbf{B}$ preferably to vanish everywhere on the source surface. In other words, the present (scalar) formulation of the heliospheric source-surface model can apply only if the magnetic field $\mathbf{B}$ is derivable from a scalar potential $V$ in the region enclosed by the source surface. If the region surrounded by the source surface were permitted to contain implicit currents, then the assumed volume of this region might reasonably be enlarged so as not to increase the overall amount of open magnetic flux. This step might help bring the solid curves in Fig. 2 into better agreement with the MHD field lines (dashed curves), which do remain significantly curved well beyond the present source surface (dotted curve). (An increase of $20-25 \%$ in source-surface volume would, for example, increase the equatorial radius $r_{0}$ in Eq. (2) from $2.33 r_{\odot}$ to $2.5 r_{\odot}$ ).

There are several levels of sophistication at which a non-spherical source surface can be used for heliospheric modeling. The trade-off is between realism and convenience. As has been noted here, the required 
algebra and numerical computations can be simplified by adopting a scalar formulation of the variational problem, minimizing Eq. (7) instead of Eq. (6) with respect to the expansion coefficients for B. However, a scalar formulation requires us to assume (as usual) that the region enclosed by the source surface is current-free, whereas comparisons of model results with various Ulysses observations cast serious doubt on this assumption. (The modeling of twisted coronal loops has long required field-aligned currents in the same region of space, but it has been hoped that such currents would be of sufficiently small scale not to influence heliospheric mappings.)

Another possible simplification is to use Eq. (2), rather than a surface of truly constant $\tilde{F}=r^{-k} \tilde{B}$, as the postulated source surface, even when $\tilde{\mathbf{B}}$ is not purely dipolar (as it never is in reality). This was the simplifying approach used by Schulz (1995) in a seemingly successful mapping of the neutral line on the source surface (see Hoeksema, 1991) to a range of heliospheric latitudes just grazed by Ulysses during Carrington Rotation 1869 (May-June 1993). If such a model source surface is Suncentered and aligned with the heliomagnetic dipole axis, then Eqs. (7) and (8) are automatically simplified through the fact that $\langle V\rangle \equiv 0$ by symmetry. [It would be possible with any source-surface geometry to make $\langle V\rangle=0$ by adding an appropriate constant to an expression such as Eq. (9), but the appropriate constant would then typically depend on the $\left\{\bar{g}_{n}^{m}, \bar{h}_{n}^{m}\right\}$. Only symmetry, such that the mean value of each sphericalharmonic function over the source surface is zero, can remove the attendant complication of Eq. (8) by making $\langle V\rangle \equiv 0$ without such an additive constant, regardless of what values are assigned to the $\left.\left\{\bar{g}_{n}^{m}, \bar{h}_{n}^{m}\right\}.\right]$

However, the use of Eq. (2) as a simplified source surface regardless of the form of $\tilde{\mathbf{B}}$ seems likely to be successful only near solar minimum, when $\tilde{\mathbf{B}}$ is in fact most nearly dipolar. The goal in choosing a nonspherical source surface is to arrange for the region(s) of minimal source-surface curvature to straddle the inner edge(s) of any heliospheric current sheet(s). The choice of a surface of constant $\tilde{F}$ as source surface shows some promise in this regard (Schulz et al., 1978; Levine et al., 1982; and in the present work) but is not guaranteed to achieve the desired configuration. However, the purpose is likely to be defeated altogether (especially near solar maximum, when $\tilde{\mathbf{B}}$ is quite complicated and multiple heliospheric current sheets are possible) if some fixed shape is assigned to the source surface, regardless of the form of $\tilde{\mathbf{B}}$.

Perhaps, however, it is really unnecessary to perfect the source-surface method as a means of modeling the heliosphere. After all, the source-surface method is only supposed to provide a simplified means of estimating results that a full three-dimensional MHD simulation of the corona and heliosphere would provide. With such MHD models already available (e.g., Usmanov, 1993; Mikic and Linker, 1994), it might make more sense to use simplified versions of source-surface models (1) to identify solar-field configurations that would be interesting to extend by MHD simulation into the helio- sphere, and (2) to set up initial (trial) field configurations that would reduce the computing time required by full MHD simulations.

Acknowledgements. This work was supported in part by JPL Contract 959569 under the Ulysses interdisciplinary scientist (IDS) program and in part by the Independent Research and Development (IR\&D) program of Lockheed Martin Missiles \& Space. The work forms part of a continuing effort inspired by scientific discussions at Science Working Team (SWT) meetings held by the Ulysses project under NASA and ESA sponsorship. The present report is based on the author's week-long poster presentation at the European Geophysical Society (EGS) meeting held 3-7 April 1995 in Hamburg, Germany.

Topical Editor R. Schwenn thanks R. J. Forsyth and another referee for their help in evaluating this paper.

\section{References}

Altschuler, M. D., and G. Newkirk, Jr., Magnetic fields and the structure of the solar corona, Solar Phys., 9, 131-149, 1969.

Hoeksema, J. T., The Solar Magnetic Field - 1985 through 1990, Report CSSA-ASTRO-91-01, Stanford University, Stanford, Calif., 1991.

Hoeksema, J. T., and P. H. Scherrer, The Solar Magnetic Field 1976 through 1985, Report UAG-94, World Data Center A for Solar-Terrestrial Physics, Boulder, Colo., 1986.

Jackson, J. D., Classical Electrodynamics, pp. 16-17, Wiley, New York, 16-17 1962.

Levine, R. H., M. Schulz, and E. N. Frazier, Simulation of the magnetic structure of the inner heliosphere by means of a non-spherical source surface, Solar Phys., 77, 363-392, 1982.

Mead, G. D., and D. H. Fairfield, A quantitative magnetospheric model derived from spacecraft magnetometer data, J. Geophys. Res., 80, 523-534, 1975.

Mikic, Z., and J. A. Linker, Disruption of coronal magnetic field arcades, Astrophys. J., 430, 898-912, 1994.

Pneuman, G. W., and R. A. Kopp, Gas-magnetic field interactions in the solar corona, Solar Phys., 18, 258-270, 1971a.

Pneuman, G. W., and R. A. Kopp, Interactions of coronal material with magnetic fields, in Solar Magnetic Fields, ed. R. Howard, pp. 526-533, Reidel, Dordrecht, 1971b.

Schatten, K. H., Current sheet magnetic model for the solar corona, in Solar Wind, eds. C. P. Sonett, P. J. Coleman, Jr., and J. M. Wilcox, pp. 44-54, NASA SP-308, Washington, D.C., 1971

Schatten, K. H., J. M. Wilcox, and N. F. Ness, A model of coronal and interplanetary magnetic fields, Solar Phys., 6, 442-455, 1969.

Schulz, M., Fourier parameters of heliospheric current sheet and their significance, Space Sci. Rev., 72, 149-152, 1995.

Schulz, M., and A. Eviatar, Diffusion of equatorial particles in the outer radiation zone, J. Geophys. Res., 74, 2182-2192, 1969.

Schulz, M., E. N. Frazier, and D. J. Boucher, Jr., Coronal magnetic-field model with non-spherical source surface, Solar Phys., 60, 83-104, 1978.

Smith, E. J., and A. Balogh, Ulysses observations of the radial magnetic field, Geophys. Res. Lett., 22, 3317-3320, 1995.

Smith, E. J., M. Neugebauer, A. Balogh, S. J. Bame, G. Erdös, R. J. Forsyth, B. E. Goldstein, J. L. Phillips, and B. T. Tsurutani, Disappearance of the heliospheric sector structure at Ulysses, Geophys. Res. Lett., 20, 2327-2330, 1993.

Stern, D. P., Representation of magnetic fields in space, Rev. Geophys. Space Phys., 14, 199-214, 1976.

Stern, D. P., The art of mapping the magnetosphere, J. Geophys. Res., 99, 17169-17198, 1994. 
Usmanov, A. V., A global numerical 3-D MHD model of the solar wind, Solar Phys., 146, 377-396, 1993.

Wang, Y.-M., and N. R. Sheeley, Jr., Solar implications of Ulysses interplanetary field measurements, Astrophys. J., 447, L143L146, 1995.
Wolfram Research, Inc., Mathematica, version 2.1, Wolfram Research, Inc., Champaign, Ill., 1992.

Zhao, X.-P., and J. T. Hoeksema, Modeling the out-of-ecliptic interplanetary magnetic field in the declining phase of Sunspot Cycle 22, Space Sci. Rev., 72, 189-192, 1995. 